\title{
Resonance phenomena in one-dimensional grating-based structures
}

\author{
I.Ya. Yaremchuk ${ }^{1}$, V.M. Fitio ${ }^{1}$, Ya.V. Bobitski ${ }^{1,2}$ \\ ${ }^{1}$ Department of Photonics, Lviv Polytechnic National University, \\ 12, Bandera str., 79013 Lviv, Ukraine \\ Phone: (8-032)-258-25-81, e-mail: iryna.y.yaremchuk@lpnu.ua \\ ${ }^{2}$ Faculty of Mathematics and Natural Sciences, \\ University of Rzeszow, Pigonia Str.1, 35959 Rzeszow, Poland
}

\begin{abstract}
Enhanced optical transmission through metallic 1-D grating-based structures has been studied using the rigorous coupled wave analysis. The results have shown that optical transmission is determined by waveguide properties of the grating slit, and there is a minimum width of slit for TE polarization, when high transmission occurs due to waveguide effect. In contrast, this limitation doesn't exist for TM polarization, and extraordinary transmission is obtained at the sub-wavelength slit. As a result, high transmission is reached due to resonance of electromagnetic field inside the grating slit.
\end{abstract}

Keywords: enhancement of optical transmission, grating, waveguide and surface plasmon-polariton resonance.

Manuscript received 15.11.16; revised version received 26.01.17; accepted for publication 01.03.17; published online 05.04.17.

\section{Introduction}

Enhanced optical transmission through grating-based structures has received growing interest among researchers due to possibility of light manipulation in a wide wavelength region. The enhancement of optical transmission through metallic periodic structure has been studied a lot of years ago [1], and still interest in such type transmission is stimulated by their applications in fields of optics and photonics. A phenomenon of enhanced optical transmission is explained by high contrast between the dielectric permittivity of metal and sub-wavelength holes [2, 3]. Moreover, the high efficiencies of transmission and enhancements of local field at certain wavelengths can be obtained using specific geometry of the metal surface [4]. The main model for studying the physical mechanism of extraordinary transmission is one-dimensional metal grating, since there TE and TM polarizations are separated $[5,6]$. There are a few theories to explain phenomenon of the extraordinary optical transmission [7-10]. In general, it is agreed that the enhanced optical transmission occurs as a result of confined surface plasmon-polariton modes at the interface of metal/dielectric. In the works $[11,12]$, it is shown that surface electromagnetic modes play a key role in appearance of resonant optical transmission. However, there are other explanations for the nature of high enhanced transmission. Presented in the works [5, 13] are the models, where transmission is modulated not by coupling to the surface plasmons but by interference of the diffracted evanescent waves, generated by subwavelength features at the surface. It was indicated [5, 14] that for lamellar transmission metallic gratings, there are two transmission resonances: one of them is coupled to the surface plasmon-polariton modes that appear on 
horizontal surfaces of the metallic grating, and the second one is related to the waveguide modes or cavities located inside the slits. In the work [15], the influence of two different mechanisms on the diffraction efficiencies waveguide and surface plasmon-polariton resonances excited in the structure was studied. However, in scientific publications one can find a lot of researches aimed at TM polarization waves and corresponding transmission spectral dependences of the grating. Simultaneously, researches regarding TE polarization can explain the anomaly of enhanced transmission in these gratings [1618]. Moreover, the elements based on this mechanism can demonstrate novel optical properties and offer new functional capabilities. The primary application of these resonant periodic structures is to design narrow-band reflection filters. Understanding the coupling of waves in metallic micro- and nanostructures has fundamental interest and practical importance in designing the optical devices that could become important elements for future micro- and nanooptical systems.

In this work, the grating-based structures transmitting $\mathrm{TM}$ and TE polarized light have been investigated. Comparison between transmissions of the TE and TM polarizations contributes to understanding the origin of enhanced optical transmission in submicron structures. Particularly, we have researched the intensity of the fields distributed inside the slit and on the surfaces of the metallic 1-D grating. This allowed us to explain nature of the resonances and to determine conditions for high transmission.

\section{Results and discussions}

The mechanism of extraordinary optical transmission through grating-based structures was studied using the rigorous coupled-wave analysis (RCWA) [19, 20]. Specifically, the RCWA and analytical transmission functions based on the same principles $[5,14,21]$ are in very good agreement with different experimental results in various geometries. The dielectric function of materials and electromagnetic fields were expanded in a Fourier series when applying the RCWA method to onedimensional grating-based structures. Infinite series of coupled equations was formed when substituting both Fourier series into Maxwell's equations. RCWA reduces the electromagnetic field calculation to an algebraic eigenvalue problem. The problem is described by the system of linear differential equations for TE and TM polarizations, respectively [22].

At the first stage of our researches, we have performed numerical calculations of transmission $(T)$ as well as sum of transmission and reflection $(T+R)$ versus the thickness of one-dimension metallic grating for the wavelength $1.5 \mu \mathrm{m}$ with the following parameters: the grating period $(\Lambda)$, width $(a)$ and height $\left(d_{m}\right)$ of the slit. The layers bounding the structure are characterized by dielectric constants $\varepsilon_{1}, \varepsilon_{3}$, groove and ridge - by $\varepsilon_{21}, \varepsilon_{22}$, correspondingly (see Fig. 1).

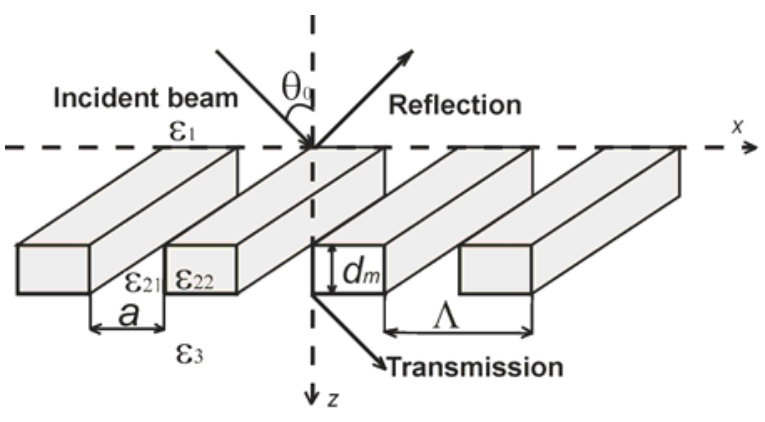

Fig. 1. Schematic structure of the grating.

The parameters of the grating for TE and TM polarization are as follows: $\Lambda=1.3 \mu \mathrm{m}, \varepsilon_{21}=9.0, a=$ $0.26 \mu \mathrm{m} ; \Lambda=1.46 \mu \mathrm{m}, \quad \varepsilon_{21}=1.0, a=0.143 \mu \mathrm{m}$ [7]. Silver (Ag) was chosen as grating material due to typical noble metal properties. The dielectric constant of silver was extrapolated using the equation $\varepsilon_{22}=4-54 \lambda^{2}+i \lambda\left(0.38+0.71 \lambda^{2}\right)$ for the wavelengths $1 \mu \mathrm{m} \leq \lambda \leq 2 \mu \mathrm{m}$. The results of calculations are presented in Fig. 2a (for TE) and in Fig. 2b (for TM). It is easy to see that high optical transmission can be obtained for both TE and TM polarizations. Fig. 2 indicates that in local maxima of transmission the reflection is low and, when the grating depth increases, then absorption increases in the grating.

At first, let us consider the case of TE polarization in detail. Fig. 3 shows the calculated transmission and sum of transmission and reflection spectra for the silver grating. The parameters used in these calculations are as follows: $\varepsilon_{21}=9.0, \quad a=0.26 \mu \mathrm{m}$ and $\Lambda=1.0 \mu \mathrm{m}$. Numbers near the curves indicate the grating depth expressed in micrometers. Fig. 3 indicates that transmission in local points decreases when the wavelength increases, and it is close to zero for the wavelengths longer than $1.8 \mu \mathrm{m}$. These facts can be explained by absence of the waveguide effect inside the slit for the wavelengths longer than $1.8 \mu \mathrm{m}$ (for $\mathrm{TE}$ polarization). Therefore, the slit represents a microresonator for a certain wavelength, which forms standing and travelling waves. This conclusion is confirmed by Fig. 3, which represents distribution of the tangential component of the modulus of electric field intensity along the coordinate $x$, i.e. along the period of grating for the grating depth $d_{m}=0.05 \mu \mathrm{m}$ (Fig. 4a) and for $d_{m}=0.2807 \mu \mathrm{m}$ (Fig. 4b) and wavelength $1.1 \mu \mathrm{m}$.

In Fig. 4, the curve 1 corresponds to the field distribution calculated at $z=0$, the curve 2 is calculated at $z=d m / 4$, curve 3 is calculated at $z=d / 2$, curve 4 is calculated at $z=3 d / 4$, and curve 5 is calculated at $z=d$, where $d$ is the slit depth. The curve 3 (Fig. 4a) describes the field distribution in the antinodes of the standing wave. The curves 2 and 4 (see Fig. 4b) correspond to the antinodes of standing wave, and the curve 3 corresponds to the node of the standing wave. 

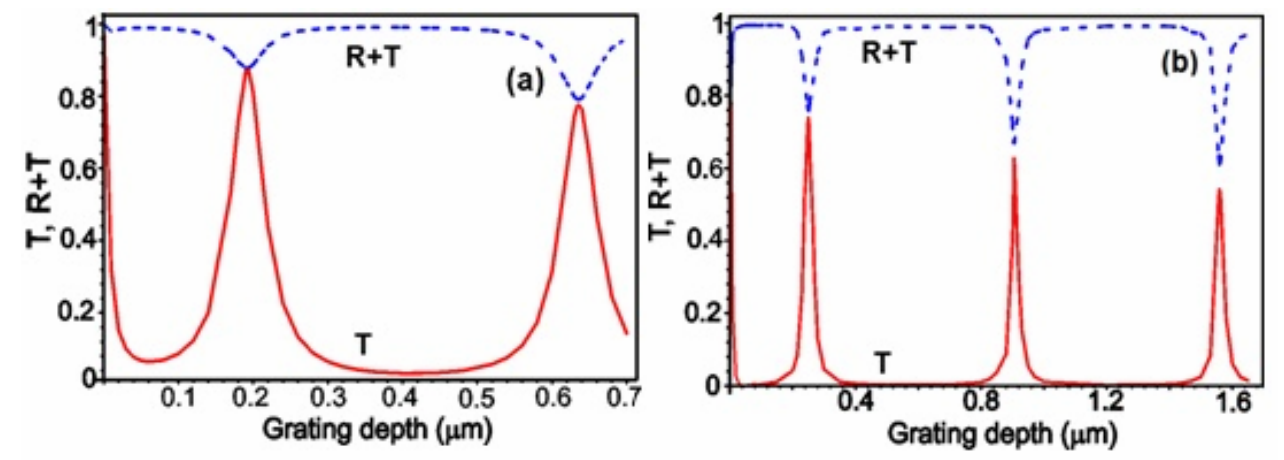

Fig. 2. Transmission and the sum of transmission and reflection spectra on the grating depth.
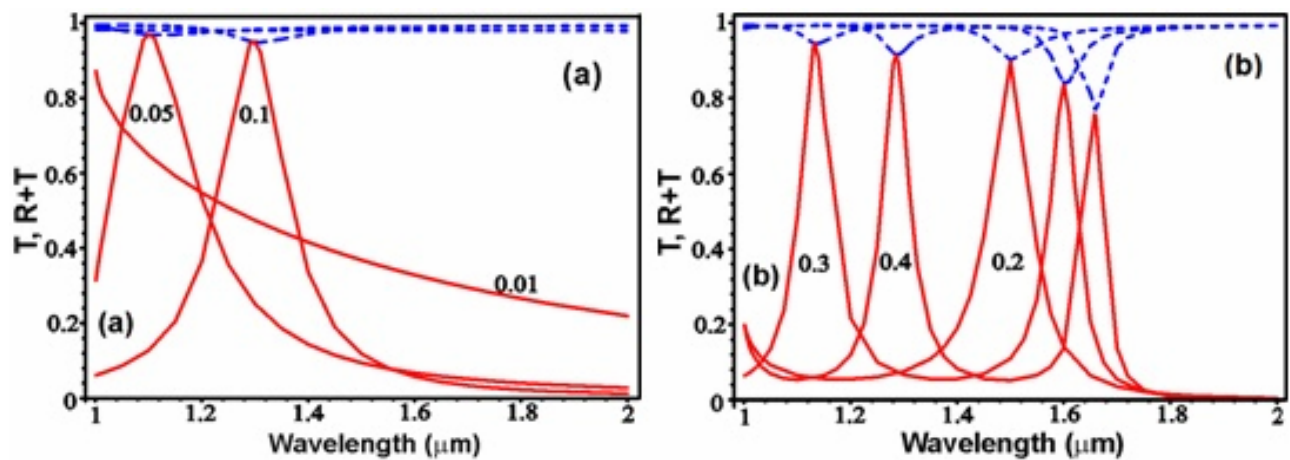

Fig. 3. Transmission, sum of transmission and reflection spectra vs. the wavelength for different grating depths at TE polarization.
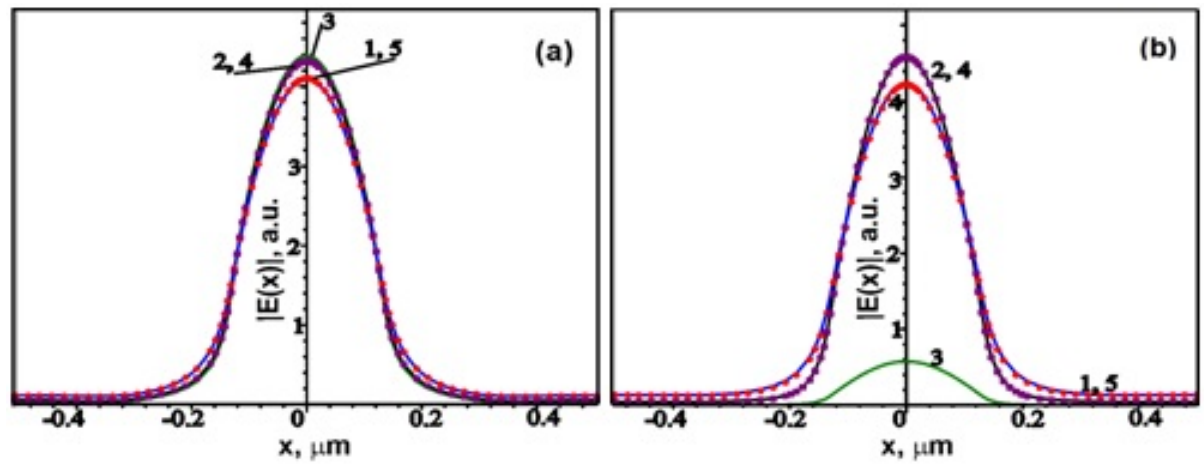

Fig. 4. Distributions of the tangential component for the modulus of electric field intensity along of the gratings period at the wavelength $1.1 \mu \mathrm{m}$ for different grating thicknesses: $0.05 \mu \mathrm{m}$ (a) and $0.2807 \mu \mathrm{m}(\mathrm{b})$, respectively.

The field intensity exceeds the unitary amplitude of the incident wave by several times within the grating slit, and it is less than unity outside the slits. Electromagnetic field is concentrated above the slit at certain grating parameters, when the resonance of field appears inside the slit. As a result, we have obtained extraordinary transmission with the simultaneous enhancement of absorption. It should be noted that modules of the field intensities are practically equal at the points that are symmetric relatively to the plane $z=d / 2$.

More detailed analysis shows that the following expression is correct:

$E_{x}(x, z) \approx(-1)^{m+1} E_{x}(x, d-z)$, where $m=1,2,3, \ldots$ As it follows from (1), for even numbers $m$ at $z=d / 2$, node of the standing wave is located inside the slit, and for odd $m$ there is an antinode of the standing wave.

In the case of $\mathrm{TM}$ polarization, spectral curves (Fig. 5) have been calculated at $\varepsilon_{21}=1.0, a=0.143 \mu \mathrm{m}$ and $\Lambda=1.0 \mu \mathrm{m}$. The numbers near the curves indicate the grating depth in micrometers. The curve with the number 0.01 represents transmission of the metallic thin film placed in medium with the refractive index 1.0 . There is only one propagation mode inside the slit unfilled with dielectric.

One can see that the transmission for the wavelength in the range of $1 \mu \mathrm{m}$ is significantly less than that for the 
longer wavelengths (e.g., at the wavelength $1.01 \mu \mathrm{m}$ for the thickness $0.05 \mu \mathrm{m}$ (see the insert in Fig. 5a). In these cases, absorption increases in the grating, and respective peaks are narrower than others. Closeness of values for these wavelengths to the grating period indicates that this effect can be caused by surface plasmon-polariton resonance on the grating surface. This fact is confirmed by the dependences of distribution inherent to the tangential component of the intensity modulus for the electric field along the coordinate $x$ for the grating with the depth $1.0 \mu \mathrm{m}$ (Fig. 6). Fig. 6a corresponds to the wavelength $1.02355 \mu \mathrm{m}$, and Fig. $6 \mathrm{~b}$ corresponds to $1.349 \mu \mathrm{m}$. The field intensity on the surface of metallic grating reaches 2 unities for the wavelength $1.02355 \mu \mathrm{m}$ causing significant surface currents and thus leading to Joule's losses. As a result, absorption in a narrow spectral range and relatively low transmission are observed. The curve 3 corresponds to the node of the standing wave, curves 2 and 4 correspond to antinodes of the standing wave for the wavelength $1.02355 \mu \mathrm{m}, \quad d_{0}=0.1108 \mu \mathrm{m}, \quad \Delta d=$ $0.4446 \mu \mathrm{m}$, and $m=2$. Therefore, two antinodes and three nodes of standing wave fit into the slit of the grating with the depth $1.0 \mu \mathrm{m}$.

The field intensity on the surface of metallic grating is significantly less than one unity for the wavelength $1.349 \mu \mathrm{m}$, and Joule's losses are negligible. Thus, surface plasmon resonance is missed, and, as a result, high transmission is observed. The curve 3 corresponds to the antinode of the standing wave, curves 2 and 4 correspond to nodes of the standing wave for this wavelength, $\mathrm{d} 0=0.41185 \mu \mathrm{m}, \Delta \mathrm{d}=0.58815 \mu \mathrm{m}$, and $\mathrm{m}=1$. These two nodes and one node of standing wave fit into the slit of the grating with the depth $1.0 \mu \mathrm{m}$.
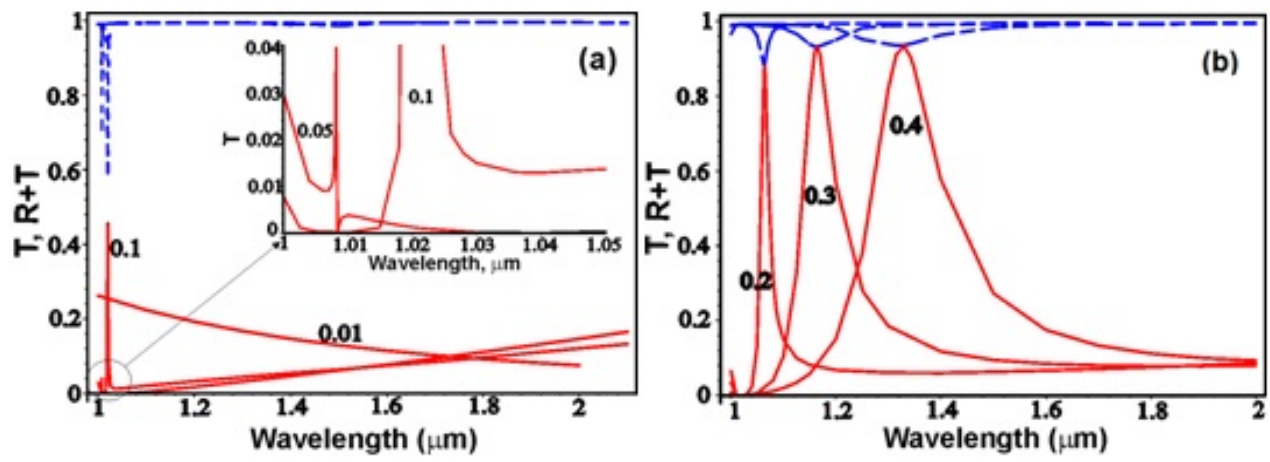

Fig. 5. Transmission, sum of transmission and reflection spectra of the silver grating with different grating depths at TM polarization.
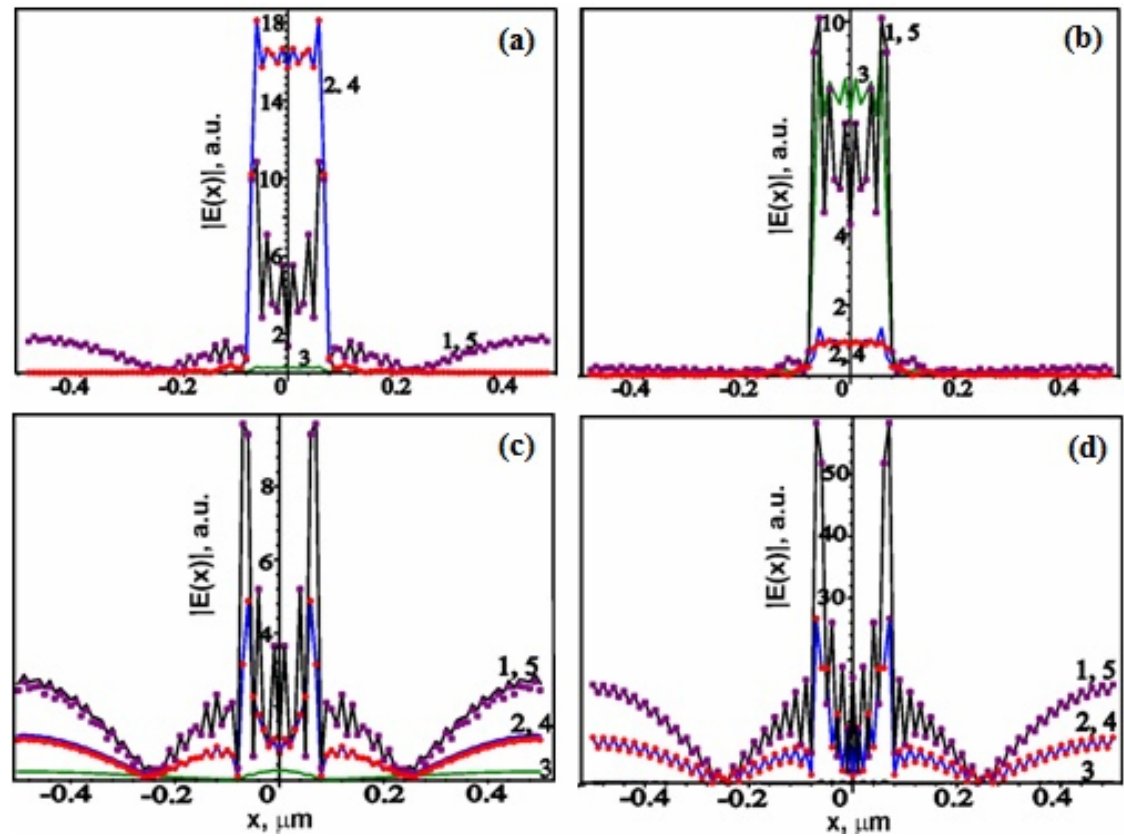

Fig. 6. Distributions of the tangential component for the electric field intensity moduli along the grating period for the grating depth $1.0 \mu \mathrm{m}$ at different wavelengths: $1.02355 \mu \mathrm{m}$ (a) and $1.349 \mu \mathrm{m}$ (b); for the grating depth $0.05 \mu \mathrm{m}$ at the wavelength $1.00816 \mu \mathrm{m}$ : (c) the imaginary part of the metal dielectric constant was taken into account in calculations; (d) imaginary part of the metal dielectric constant was neglected. 
The modules of the field intensities at the points symmetric relatively to the plane $z=d / 2$ are equal (like to the case of TE polarization). More detailed analysis shows that for diffraction wave of TM polarization by the binary metallic grating the following expression can be applied:

$$
E_{x}(x, z) \approx(-1)^{m} E_{x}(x, d-z),
$$

where $m=1,2,3, \ldots$ As it follows from (2), for odd numbers $m$ at $z=d / 2$, the node of the standing wave lies inside the slit, and for even $m$ there is an antinode of the standing wave, i.e. the situation is opposite to the case of TE polarization. Eqs. (1) and (2) indicate that for even $m$ the field distribution by grating at TE polarization describes the sum of both the symmetric and asymmetric functions relatively to the axis $z$. It should be noted that the value of symmetric function is much less than that of the asymmetric one at TE polarization, and vice versa for TM one. The situation is opposite for odd $m$. This conclusion was made on the base of numerical calculations of the field distribution inside the slit and on the grating surface. According to these calculations, if we move inside the slit from $z=0$ (if slit is long enough), the first will be the antinode of standing wave, then the second node of standing wave, then again antinode etc. for TE polarization. The situation is opposite for TM polarization.

One can conclude that the plasmon resonance on grating surface results in Joule's losses and low transmission. This conclusion is confirmed by Figs. 6c and $6 \mathrm{~d}$, which shows distribution of the intensity modulus for electric field along the grating period for the following parameters: wavelength $1.00816 \mu \mathrm{m}$, grating depth $0.05 \mu \mathrm{m}$. Fig. $6 \mathrm{c}$ shows the results when the imaginary part of the dielectric function for silver was taken into account. The calculation results, in case when imaginary part of the metal dielectric constant is negligible, are presented in Fig. 6d. Transmission of this grating for actual metal is 0.04 for the wavelength $1.00816 \mu \mathrm{m}$ (see Fig. 5a). Significant field intensities ( 3 a.u.) are on the grating surfaces, thus there the plasmon resonance takes place (Fig. 6c).

One can expect significant Joule's losses in the case when imaginary part of the metal dielectric constant is taken into account. In case of neglected imaginary part of dielectric constant of metal, fields are increased on grating boundaries with homogeneous media to 20 a.u. and inside the slit to 50 a.u. Thus, the plasmon resonance takes place both at the grating surface and in the slit. Transmission of this grating equals to unity for the wavelength $1.00816 \mu \mathrm{m}$. Both gratings have different characteristics at the wavelength $1.00816 \mu \mathrm{m}$ with increasing the depth. Transmission of the grating with losses is less than 0.04 ; transmission of the grating without losses is equal to unity at the resonance depths $d_{0}=0.05 \mu \mathrm{m}, d_{1}=0.473 \mu \mathrm{m}$, and $d_{2}=0.911 \mu \mathrm{m}$. In fact, our numerical calculations show that transmission is higher than 0.997 for all the analyzed depths. The grating without losses has the propagation constant $\beta=7.1755 \mu \mathrm{m}^{-1}$ and, correspondingly, $\Delta d=\pi / \beta=0.438 \mu \mathrm{m}$.

\section{Conclusions}

The transmission and reflection of the periodic structures based on the metallic grating have been investigated. The resonant transmission for TM polarization is achieved due to the waveguide effect and excitation of plasmon-polaritons on metallic surfaces due to metallic grating that makes a slight perturbation in the plane of the metal. The absorption increases in the grating as a result of the plasmon resonance, and thus the transmittance is reduced. The waveguide effect inside the slit is possible for any width of slit, and thus it can provide abnormally high transmittance. The resonant transmission for TE polarization only occurs due to the waveguide effect, and there is a minimum width of slit, when the waveguide effect and, consequently, high transmission are possible. The metallic grating period and thickness, refractive indices of grating and grating slit are the main parameters that control the peak positions and transmission amplitudes. This structure can be used in the design of selective transmission narrow-band filter for an infrared region.

\section{Acknowledgments}

The work was supported by Ministry of Education and Science of Ukraine (grant DB $\backslash$ Tekton No. 0115U000427).

\section{References}

1. Ebbesen T.W., Lezec H.J., Ghaemi H.F., Thio T., Wolff P.A. Extraordinary optical transmission through sub-wavelength hole arrays. Nature. 1998. 391(6668). P. 667-669.

2. Karabchevsky A., Krasnykov O., Abdulhalim I., Hadad B., Goldner A., Auslender M., Hava S. Metal grating on a substrate nanostructure for sensor applications. Photonics and Nanostructures - Fundamentals and Applications. 2009. 7, No. 4. P. 170-175.

3. Lindquist N.C., Nagpal P., McPeak K.M., Norris D.J., Oh S.H. Engineering metallic nanostructures for plasmonics and nanophotonics. Repts. Progr. Phys. 2012. 75, No. 3. P. 036501.

4. De Ceglia D., Vincenti M.A., Scalora M., Akozbek N., Bloemer M. Plasmonic band edge effects on the transmission properties of metal grating. AIP Adv. 2011. 1. P. 032151-1-032151-15.

5. Cao Q., Lalanne P. Negative role of surface plasmons in the transmission of metallic gratings with very narrow slits. Phys. Rev. Lett. 2002. 88, No. 5. P. 057403. 
6. Porto J.A., Garcia-Vidal F.J., Pendry J.B. Transmission resonances on metallic gratings with very narrow slits. Phys. Rev. Lett. 1999. 83, No. 14. P. 2845.

7. Treacy M.M.J. Dynamical diffraction explanation of the anomalous transmission of light through metallic gratings. Phys. Rev. B. 2002. 66, No. 19. P. 195105.

8. Barbara A., Quémerais P., Bustarret E., Lopez-Rios T. Optical transmission through subwavelength metallic gratings. Phys. Rev. B. 2002. 66, No. 16. P. 161403.

9. Martin-Moreno L., Garcia-Vidal F.J., Lezec H.J., Pellerin K.M., Thio T., Pendry J.B., Ebbesen T.W. Theory of extraordinary optical transmission through subwavelength hole arrays. Phys. Rev. Lett. 2001. 86, No. 6. P.1114.

10. Yao N., Pu M., Hu C., Lai Z.A., Zhao Z., Luo X. Dynamical modulating the directional excitation of surface plasmons sources. Optik - Intern. Journal for Light and Electron Optics. 2012. 123, No. 16. P. 1465-1468.

11. Liu H., Lalanne P. Microscopic theory of the extraordinary optical transmission. Nature. 2008. 452(7188). P. 728-731.

12. Garcia-Vidal F.J., Martin-Moreno L., Ebbesen T.W., Kuipers L. Light passing through subwavelength apertures. Rev. Mod. Phys. 2010. 82, No. 1. P. 729.

13. Lezec H., Thio T. Diffracted evanescent wave model for enhanced and suppressed optical transmission through subwavelength hole arrays. Opt. Exp. 2004. 12, No. 16. P. 3629-3651.

14. Xie S., Li H., Fu S., Xu H., Zhou X., Liu Z. The extraordinary optical transmission through double- layer gold slit arrays. Opt. Communs. 2010. 283. P. 4017-4024.

15. Yaremchuk I.Y., Fitio V.M., and Bobitski Y.V. High transmission of light through metallic grating limited by dielectric layers. LFNM'2013. 2013. P. 74.

16. Skigin D.C., Depine R.A., Resonances on metallic compound transmission gratings with subwavelength wires and slits. Opt. Communs. 2006. 262. P. 270-275.

17. Fitio V.M. Transmissions of Metallic Gratings with Narrow Slots. 2006 International Workshop on Laser and Fiber-Optical Networks Modeling, June 29 - July 1, 2006. P. 113-116.

18. Moreno E., Martín-Moreno L., García-Vidal F.J. Extraordinary optical transmission without plasmons: the s-polarization case. J. Opt. A: Pure and Appl. Opt. 2006. 8, No. 4. P. S94.

19. Moharam M.G., Gaylord T.K. Rigorous coupledwave analysis of planar-grating diffraction. JOSA, 1981. 71, No. 7. P. 811-818.

20. Moharam M.G., Gaylord T.K., Grann E.B., Pommet D.A. Formulation for stable and efficient implementation of the rigorous coupled-wave analysis of binary gratings. JOSA A. 1995. 12, No. 5. P. 1068-1076.

21. Benabbas A., Halte V., Bigot J. Analytical model of the optical response of periodically structured metallic films. Opt. Exp. 2005. 13. P. 8730-8745.

22. Yaremchuk I., Tamulevičius T., Fitio V., Gražulevičiūte I., Bobitski Ya., Tamulevičius S. Numerical implementation of the S-matrix algorithm for modeling of relief diffraction gratings. J. Mod. Opt. 2013. 60. P. 1781-1788. 